\title{
High expression levels of CLEC4M indicate poor prognosis in patients with hepatocellular carcinoma
}

\author{
LIUPING LUO ${ }^{1,2}$, LIHONG CHEN ${ }^{2}$, KUN KE $^{1,2}$, BIXING ZHAO $^{2}$, LILI WANG $^{3}$, CUILIN ZHANG $^{2}$, FEI WANG $^{2}$, \\ NAISHUN LIAO ${ }^{2}$, XIAOYUAN ZHENG ${ }^{2}$, XIAOLONG LIU ${ }^{2}$, YINGCHAO WANG $^{2}$ and JINGFENG LIU ${ }^{2}$ \\ ${ }^{1}$ The First Affiliated Hospital of Fujian Medical University, Fuzhou, Fujian 350005; \\ ${ }^{2}$ The United Innovation of Mengchao Hepatobiliary Technology Key Laboratory of Fujian Province, \\ Mengchao Hepatobiliary Hospital of Fujian Medical University, Fuzhou, Fujian 350025; \\ ${ }^{3}$ Department of Diagnostic Radiology, Fujian Medical University Union Hospital, Fuzhou, Fujian 350001, P.R. China
}

Received January 7, 2019; Accepted November 12, 2019

DOI: $10.3892 / \mathrm{ol} .2020 .11294$

\begin{abstract}
The identification of novel and accurate biomarkers is important to improve the prognosis of patients with hepatocellular carcinoma (HCC). C-Type lectin domain family 4 member $\mathrm{M}$ (CLEC4M) is involved in the progression of numerous cancer types. However, the clinical significance of CLEC4M in HCC is yet to be elucidated. The aim of the present study is to evaluate the involvement of CLEC4M in HCC progression. The expression level of CLEC4M was determined in tumor, and their corresponding adjacent non-tumor tissues derived from 88 patients with HCC, using immunohistochemistry, western blot and reverse transcription-quantitative PCR. The correlation between CLEC4M expression and certain clinicopathological characteristics was retrospectively analyzed. The results suggested that CLEC4M was specifically labeled in sinusoidal endothelial cells, in both HCC and non-tumor tissues. Moreover, the expression of CLEC4M in tumor tissues was significantly lower than that in non-tumor tissues $(\mathrm{P}<0.0001)$, which indicated its potential as a biomarker of the development of HCC. Subsequently, correlation analysis suggested that the relatively higher CLEC4M expression in HCC tissues was significantly associated with increased microvascular invasion $(\mathrm{P}=0.008)$, larger tumor
\end{abstract}

Correspondence to: Professor Jingfeng Liu or Dr Yingchao Wang, The United Innovation of Mengchao Hepatobiliary Technology Key Laboratory of Fujian Province, Mengchao Hepatobiliary Hospital of Fujian Medical University, 312 Xihong Road, Fuzhou, Fujian 350025, P.R. China

E-mail: drjingfeng@126.com

E-mail: yingchaowang@live.com

Abbreviations: HCC, hepatocellular carcinoma; CLEC4M, C-type lectin domain family 4 member M; RFS, recurrence free survival; OS, overall survival; RT-qPCR, reverse transcription-quantitative polymerase chain reaction

Key words: HCC, CLEC4M, recurrence, metastasis, prognostic factor size $(\mathrm{P}=0.018)$, absence of tumor encapsulation $(\mathrm{P}<0.0001)$ and lower tumor differentiation $(\mathrm{P}=0.019)$. Notably, patients with high CLEC4M expression levels in their tumor tissues experienced more frequent recurrence and shorter overall survival (OS) times compared with the low-expression group. Furthermore, CLEC4M expression in tumor tissues was identified as an independent and significant risk factor for recurrence-free survival and OS. The results of the present study suggest that CLEC4M may be a valuable biomarker for the prognosis of the patients with HCC, postoperatively.

\section{Introduction}

Hepatocellular carcinoma (HCC) is the sixth most prevalent malignant cancer type, and results in $\sim 800,000$ fatalities each year, making it the fourth leading cause of cancer-associated mortality worldwide (1). In China, HCC is commonly treated using surgical resection combined with either chemo- or radiotherapy, and immunotherapy is used less frequently (2). Despite the current therapeutic strategies achieving promising results in certain cases, it is reported that the 5-year overall and disease-free survival rates are just 51 and $27 \%$, respectively (3). High rates of tumor recurrence and metastasis are significant barriers to further improving HCC treatment, thus, early detection is crucial for successful treatment. Numerous HCC biomarkers (such as $\alpha$-fetoprotein, cancer antigen 19-9 and glypican 3 ) have been identified and clinically applied to improve the diagnosis of HCC. However, due to their low sensitivity and specificity, the diagnostic accuracy of these biomarkers is insufficient (4-6). Therefore, it is necessary to further elucidate the underlying mechanisms of HCC recurrence and metastasis, and to identify novel biomarkers for the early diagnosis of HCC.

The C-type lectins comprise a large superfamily of proteins, including selectins, mannose receptors (MRs) and liver and lymph node sinusoidal endothelial cell C-type lectin (CLEC4G) (7). The C-type lectins possess several biological functions, such as mediation of the inflammatory and immune responses (8), and pathogen recognition using shared homologous carbohydrate-recognition domains. Increasing evidence has suggested that the dysregulation of C-type lectins is closely 
associated with the progression of cancer. For example, serum E-selectin expression was significantly higher in patients with colorectal cancer compared with healthy subjects or patients with benign colorectal diseases (9). Similarly, CLEC18B expression was significantly altered in glioblastoma multiforme tissue, and was identified as an independent predictor of patient survival (10). Additionally, it has been demonstrated that selectin influences the progression of numerous cancer types, including colon carcinoma $(11,12)$ and gastric cancer (13). Coupland et al (14) discovered that P-selectin promoted the lung metastasis of breast cancer and melanoma in vivo. Moreover, the interaction between E-selectin and its ligands enhanced the adhesion of prostate cancer cells to endothelial cells (15).

C-Type lectin domain family 4 member M, (CLEC4M; also known as CD209L) belongs to the C-type lectin family and is expressed in the endothelial cells of the lymph nodes and liver. It exhibits $\sim 80 \%$ amino acid homology with dendritic cell-specific intercellular adhesion molecule-3-grabbing non-integrin (DC-SIGN) (16). Unlike CLEC4M, DC-SIGN is expressed on the surface of dendritic cells and a subset of macrophages (17). It has been demonstrated that cell surface expression of CLEC4M promotes HIV (18), and hepatitis C (19) and hepatitis B (20) infection by increasing contact between the virus and host cell. Several studies have demonstrated the involvement of CLEC4M in colon and gastric cancer progression. For example, CLEC4M is associated with poor prognosis in patients with colon cancer, and it is involved in the adhesion, migration, invasion and liver metastasis of colon cancer cells (21). Furthermore, CLEC4M exerts these biological functions through the signal transducer and activator of transcription 5/long non-coding RNA Heterogeneous Nuclear Ribonucleoprotein K Pseudogene 2/ C-X-C chemokine receptor type 4 axis (13). Although CLEC4M has been examined in several malignant tumor types, to our knowledge, the clinical significance of CLEC4M has never been investigated in HCC so far.

\section{Materials and methods}

Patient information and specimen collection. Fresh frozen tissues and pathological sections were collected (in a double-blind manner) from 88 patients (82 males and 6 females) with HCC, who had completed a follow-up survey at the Mengchao Hepatobiliary Hospital of Fujian Medical University (Fujian, China) between December 2013 and July 2016. The ages of enrolled patients range from 34 to 71 years (median age, 54 years). The expression level of CLEC4M was detected using reverse transcription-quantitative PCR (RT-qPCR) and immunohistochemical staining. Additionally, 18 distant non-tumor hemangioma tissues were collected, and the expression level of CLEC4M was determined and used as a healthy hepatic tissue control. All of the enrolled patients exhibited preoperative liver function of Child-Pugh score A or B (without distant metastasis), and a follow-up was conducted every 6 months via telephone or outpatient service, until July 2017. Western blotting was conducted on a total of 9 fresh frozen HCC tissue samples (from the cohort of 88 patients as aforementioned), in order to examine the differences in the expression level of CLEC4M between patients in the recurrence/metastasis group ( $\mathrm{R} / \mathrm{M}$ group) and the no R/M group (N R/M group), according to their prognosis after surgery. The use of human tissue samples in the present retrospective study was approved by the ethics committee of Mengchao Hepatobiliary Hospital of Fujian Medical University (Fujian, China) and written informed consent was preoperatively obtained from each participant.

Immunohistochemical detection of CLEC4M. Immunohistochemical analysis was performed to determine the expression level of CLEC4M in 88 pathological sections as previously described (22) with slight modification. Briefly, the $4-\mu \mathrm{m}$ sections were deparaffinized in xylene and rehydrated in graded ethanol. Afterwards, the slides were blocked with $1 \%$ $\mathrm{H}_{2} \mathrm{O}_{2}$ for $30 \mathrm{~min}$ at room temperature. Following rehydration, the slides were incubated with EDTA (0.1 M, pH 9.0) antigen repairing buffer in a high-pressure cooker and heated until steam was generated. The slides were further incubated for $3 \mathrm{~min}$, and then cooled off at room temperature. Thereafter, the slides were probed with a rabbit anti-CLEC4M antibody (1:800; cat. no. ab169783; Abcam) or mouse anti-CD31 antibody (1:200; cat. no. 3528S; CST Biological Reagents Co., Ltd.) overnight at $4^{\circ} \mathrm{C}$. Then, a commercial immunohistochemical staining kit (cat. no. KIT-5020; Fuzhou Maixin Biotech Co., Ltd.), alexa 488-conjugated anti-rabbit secondary antibody against CLEC4M (1:1,000; cat. no. A-11008; Thermo Fisher Scientific, Inc.) and alexa 546-conjugated anti-mouse secondary antibody against CD31 (1:1,000; cat. no. A-11003; Thermo Fisher Scientific, Inc.) were used to incubate the slides respectively, as the manufacturer's instructions. The immunohistochemical score was based on the international German immune response scoring system (German immunoreactive score, IRS) (23) according to the intensity of staining; two pathologists, who were blinded to the clinical characteristics of the specimens, independently performed the scoring. The final score for each section was based on the mean of the scores obtained by the two pathologists, and were graded as follows: 0 , negative; $1+$, weak positive; $2+$, moderate positive; or $3+$, strong positive.

RNA extraction and RT-qPCR. The expression level of CLEC4M mRNA in 88 fresh frozen tumorous tissues, and their corresponding adjacent non-tumor tissues, was calculated. Total RNA was extracted from tissues using the TransZol up plus kit (Beijing Transgen Biotech Co., Ltd.) according to the manufacturer's instructions. Following quantification, $1 \mu \mathrm{g}$ total RNA from each sample was reverse-transcribed into cDNA using the Transcriptor First Strand cDNA Synthesis Kit (Roche Diagnostics; cat. no. 04897030001) according to the manufacturer's protocol. Subsequently, qPCR detection was carried out under the following conditions: 40 cycles of $95^{\circ} \mathrm{C}$ for $10 \mathrm{sec}, 60^{\circ} \mathrm{C}$ for $60 \mathrm{sec}$ and a final extension for $30 \mathrm{sec}$ at $72^{\circ} \mathrm{C}$. All PCR reactions were repeated $\geq 3$ times. $\beta$-actin was selected as the endogenous control and the primer pairs are detailed in Table I. The relative gene expression of CLEC4M was calculated using the $2^{-\Delta \Delta \mathrm{Cq}}$ method (24).

Western blotting. Total protein was extracted from 9 HCC tissue samples using RIPA lysis buffer (Beijing Solarbio Science \& Technology Co., Ltd.) supplemented with a protease 
Table I. Primer sequences used in RT-qPCR

\begin{tabular}{lc}
\hline Gene name & \multicolumn{1}{c}{ Primer sequence } \\
\hline CLEC4M-Forward & 5'-TGGGCCTCCTGGAAGAAGAT-3' \\
CLEC4M-Reverse & 5'-GCGTCTTGCTCGGATTGTTC-3' \\
$\beta$-actin-Forward & 5'-GCCAACACAGTGCTGTCTGG-3' \\
$\beta$-actin-Reverse & 5'-GCTCAGGAGCAATGATCTTG-3'
\end{tabular}

CLEC4M, C-type lectin domain family 4 member M.

inhibitor cocktail (cat. no. 11697498001; Roche Diagnostics). The protein was then quantified using a bicinchoninic acid protein assay kit (Beijing Solarbio Science \& Technology Co., Ltd.) according to the manufacturer's instructions. Equal amounts $(50 \mu \mathrm{g})$ of protein were separated via SDS-PAGE on a $12 \%$ gel, and transferred to PVDF membranes. Subsequently, the membranes containing the separated proteins were blocked with 5\% BSA for $2 \mathrm{~h}$ at room temperature. Next, the membranes were probed with primary antibodies against CLEC4M (1:1,000; cat. no. \#ab169783; Abcam) overnight at $4^{\circ} \mathrm{C}$. The membranes were then incubated with horseradish peroxidase-conjugated secondary antibodies (1:2,000 dilution; cat. no. HS201-01; Beijing Transgen Biotech Co., Ltd.) for $1 \mathrm{~h}$ at room temperature. Protein bands were visualized using an electrochemical luminescence reagent (Thermo Fisher Scientific, Inc). $\beta$-actin (anti- $\beta$-actin antibody; $1: 1,000$; cat. no. sc-47778; Santa Cruz Biotechnology, Inc.) was used as an endogenous control. Densitometry of blot was analyzed by Image $\mathbf{J}$ software (version 1.45). Protein expression was subsequently quantified with densitometry and normalized by $\beta$-actin.

Bioinformatics analysis. The expression of CLEC4M was also investigated in a transcriptome dataset of our lab and The Cancer Genome Atlas (TCGA) database (https://www. cancer.gov/tcga). The transcriptome dataset included whole RNA-sequencing data of tumor and non-tumor tissue samples collected from $65 \mathrm{HCC}$ patients receiving surgical resection in Mengchao Hepatobiliary Hospital of Fujian Medical University. After mapping to reference genome (GRCh37), annotation and quantification of gene expression were conducted with STAR (version 2.5.3a) as previously described (25). On the other hand, the mRNA sequencing (mRNA-Seq) expression spectrum data (HiSeqV2), including $371 \mathrm{HCC}$ patients was downloaded from the UCSC cancer browser (https://genome-cancer.ucsc. edu) as previously described (26). The expression data $(n=50)$ available for both primary HCC tissues and paired non-tumor in TCGA dataset were extracted to analyze the expression of CLEC4M.

Statistical analysis. Statistical analyses were conducted using SPSS (V20.0, IBM Corp.) or Prism statistical software (GraphPad Software, Inc.). The data were presented as the mean \pm SD. Comparisons between two groups were performed using the two-tailed paired or unpaired Student's t-test. One-way ANOVA was used for comparisons between groups, followed by Tukey's multiple comparisons test as the post-hoc test. The $\chi^{2}$-test and/or the Fisher's exact test were conducted to analyze the association between CLEC4M expression level and various clinicopathological features of patients with HCC. The Cox proportional hazard model was used to analyze univariate and multivariate survival time data. Kaplan-Meier analysis and the Log-rank test were used to analyze the OS times of patients with HCC. $P<0.05$ was considered to indicate a statistically significant difference.

\section{Results}

CLEC4M expression is significantly downregulated in HCC tissues. In a previous study, isobaric tags were used for the relative and absolute quantitation of coupling, alongside 2-D liquid chromatography-tandem mass spectrometry, to analyze the proteomic differences between HCC and non-tumor tissues (data unpublished). It was subsequently, determined that CLEC4M expression was downregulated in HCC tumor tissues. To confirm these proteomics results, RT-qPCR was conducted to detect the mRNA expression level of CLEC4M in 88 pairs of tumor tissues and their corresponding adjacent non-tumor tissues. As exhibited in Fig. 1A, the expression of CLEC4M was significantly decreased in tumor tissues compared with paratumor tissues $(\mathrm{P}<0.0001)$. Furthermore, the aforementioned results were supported by analysis of the datasets retrieved from our transcriptome database $(\mathrm{P}<0.0001$; Fig. 1B) and TCGA dataset ( $\mathrm{P}<0.0001$; Fig. 1C).

To investigate the clinical significance of CLEC4M, its expression level in paraffin-embedded HCC samples $(n=88)$ was compared with normal hepatic tissue samples $(n=18)$ using RT-qPCR. The representative images of HCC tissues with negative, weakly positive, moderate-positive and strong-positive CLEC4M staining are exhibited in Fig. 1D. The pathological results indicated that the expression level of CLEC4M in both paratumor tissue and distant hepatic tissue is significantly higher than that in HCC tissue $(\mathrm{P}<0.0001$; Figs. 1E and 2A). Moreover, RT-qPCR conducted on another group of $52 \mathrm{HCC}$ tissue samples, 27 paratumor tissue samples and 18 normal hepatic samples supported the hypothesis that the expression level of CLEC4M in HCC tissues is significantly lower compared with paratumor and normal hepatic tissues (Fig. 1F).

The association between CLEC4M expression level and various clinicopathological characteristics of HCC. The correlation between CLEC4M protein expression and various clinicopathological characteristics was investigated. The data of multiple demographic parameters (including sex, age, pathological diagnosis and certain tumor characteristics), as well as follow-up data, were collected and recorded. The median follow-up period was 34 months (range, 5-47 months). Subsequent association analysis determined that high expression levels of CLEC4M in HCC tissues significantly correlated with larger tumor size $(\mathrm{P}=0.018)$, none encapsulation $(\mathrm{P}=0.0006)$, the presence of microvascular invasion $(\mathrm{P}=0.008)$, and increased primary differentiation $(\mathrm{P}=0.019)$. However, there was no significant correlation between high CLEC4M expression levels and advanced Barcelona clinic liver cancer stage, and $\alpha$-fetoprotein (AFP) expression, when the samples were divided into two groups, including: 
A

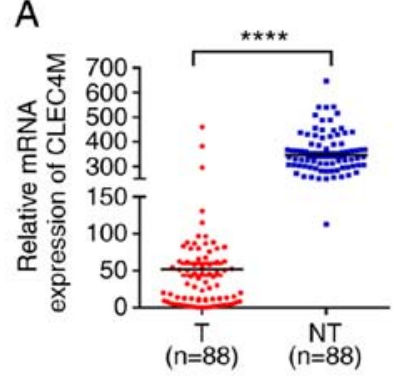

B

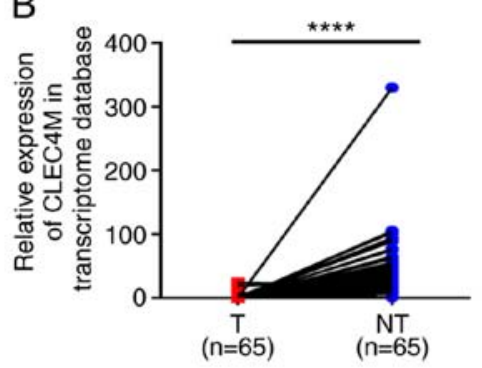

Weak Moderate

Negative
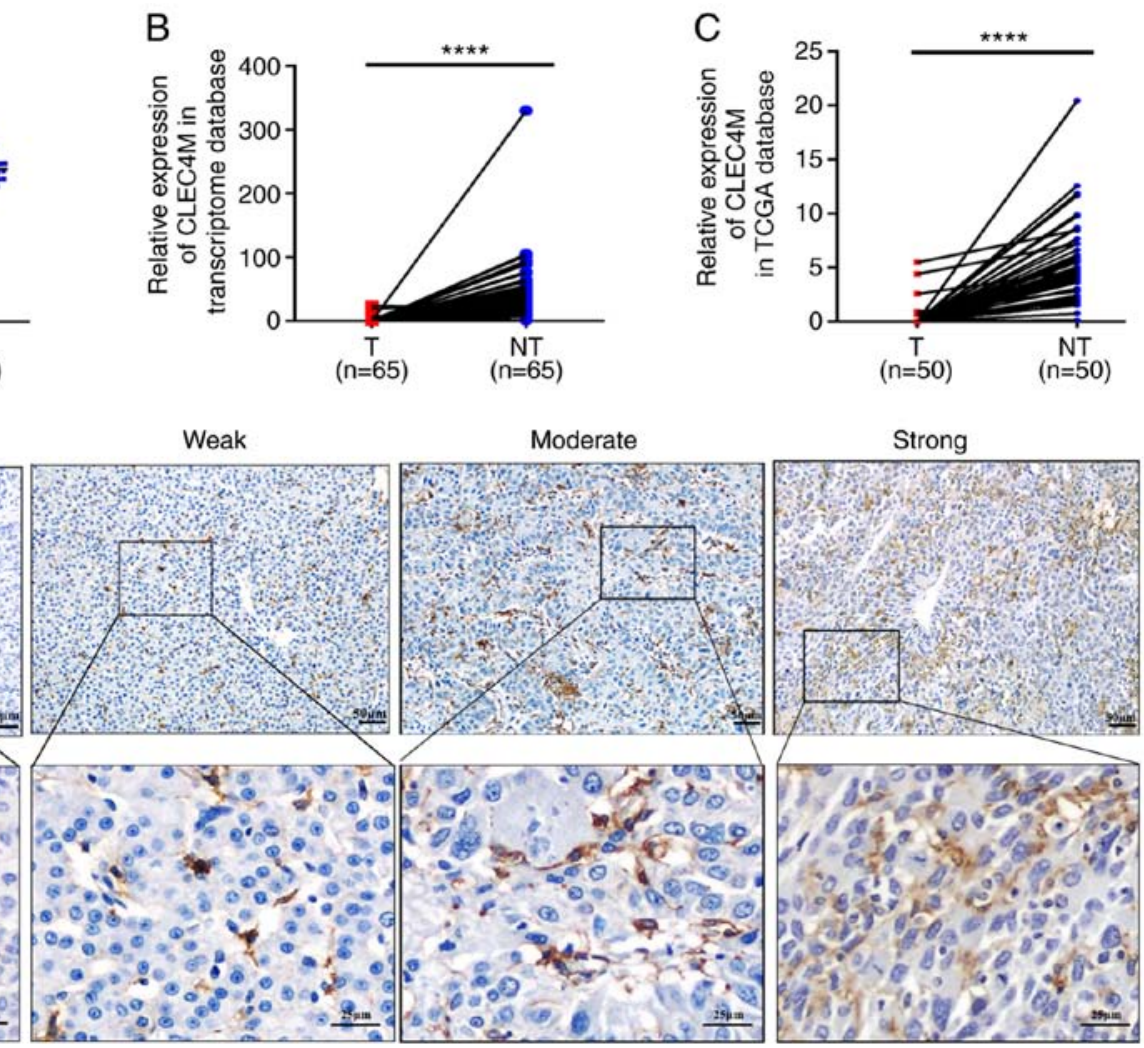

E
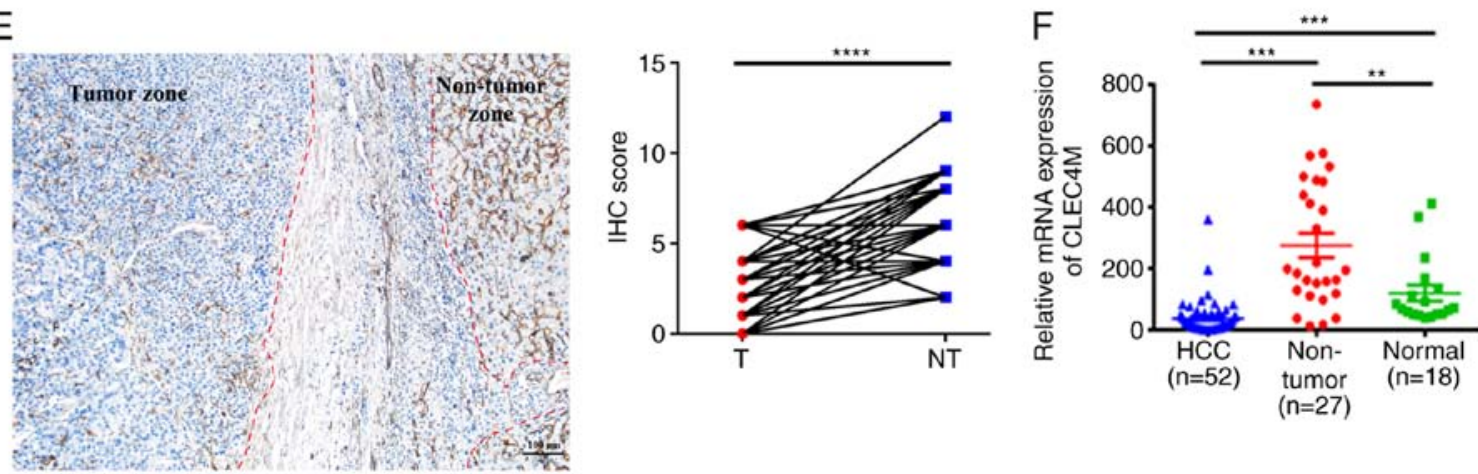

Figure 1. Determination of CLEC4M expression level in HCC tumor vs. adjacent non-tumor tissues. (A) RT-qPCR results of CLEC4M mRNA expression in 88 pairs of HCC tumor samples and their corresponding adjacent non-tumor tissues; paired Student's t-test. (B) transcriptomic analysis of the relative expression of CLEC4M in HCC tissues vs. adjacent non-tumor tissues. N=65, paired Student's t-test. (C) Analysis of CLEC4M mRNA expression in The Cancer Genome Atlas database; $\mathrm{n}=50$; paired t-test. (D) CLEC4M expression levels in HCC tumor tissues. CLEC4M expression was semi-quantitatively divided into four groups: negative staining, weak-positive staining, moderate-positive staining and strong-positive staining (magnification, $\mathrm{x} 40$ and $\mathrm{x} 200$ ); (E) representative immunohistochemical section of HCC border indicating the decreased CLEC4M expression in the tumor tissue (magnification, $\mathrm{x} 200$; (F) RT-qPCR results of CLEC4M mRNA expression in HCC ( $\mathrm{n}=52)$, non-tumor tissues $(\mathrm{n}=27)$ and normal hepatic tissues (n=18); One-way ANOVA combined with Tukey's multiple comparisons test (post-hoc) was applied to perform the comparisons between groups. ${ }^{* *} \mathrm{P}<0.01,{ }^{* * * *} \mathrm{P}<0.001$ and ${ }^{* * * *} \mathrm{P}<0.0001$. T, tumor tissue; $\mathrm{NT}$, non-tumor tissue. HCC, hepatocellular carcinoma; CLEC4M, C-type lectin domain family 4 member M; RFS, recurrence free survival; RT-qPCR, reverse transcription-quantitative polymerase chain reaction; IHC, immunohistochemical.

negative and weak $(n=59)$; and moderate and strong $(n=29)$, as summarized in Table II. The results of the present study indicate that abnormal expression levels of CLEC4M may be associated with the recurrence and metastasis of HCC. Hence, the RT-qPCR data were further divided into two groups (the $\mathrm{N}$ R/M group and the R/M group), depending on whether the $\mathrm{HCC}$ had recurred or metastasized. The results suggested that CLEC4M staining in the $\mathrm{R} / \mathrm{M}$ group was dramatically stronger compared with that of the $\mathrm{N} \mathrm{R/M}$ group, but weaker than that in non-tumor tissue and normal tissue (Fig. 2A). Furthermore, high expression of CLEC4M in tumor tissue was positively associated with the recurrence and metastasis of HCC $(3 * 2$ $\chi^{2}$ test, $\mathrm{P}=0.004$, Table III), which was further confirmed by RT-qPCR analysis ( $\mathrm{P}=0.035$; Fig. $2 \mathrm{~B})$ and western blotting $(\mathrm{P}=0.049$; Fig. 2C).

High CLEC4M expression levels in HCC tissues indicate poor prognosis in patients with HCC. The influence of CLEC4M expression on recurrence free survival (RFS) and overall survival (OS) time was then investigated using Kaplan-Meier analysis. The RFS and OS times were significantly lower in patients with a high expression level of CLEC4M, compared with those with low expression levels $(\mathrm{P}=0.0001$ and $\mathrm{P}=0.0011$, respectively; Fig. 3A and B). The mean RFS time was 14 months 
A

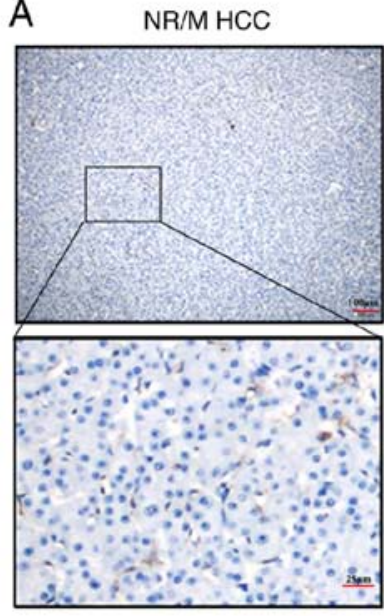

B

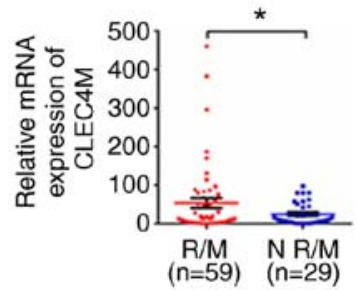

$\mathrm{R} / \mathrm{M} \mathrm{HCC}$

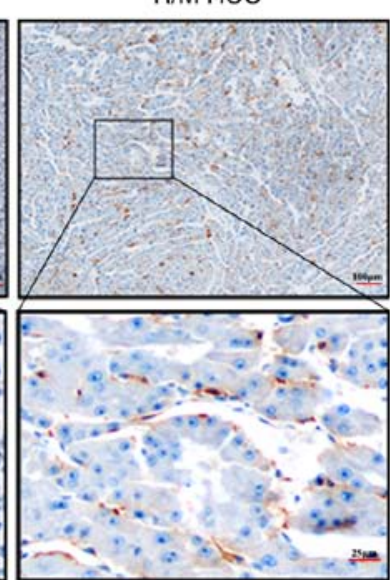

Adjacent non-tumor tissue

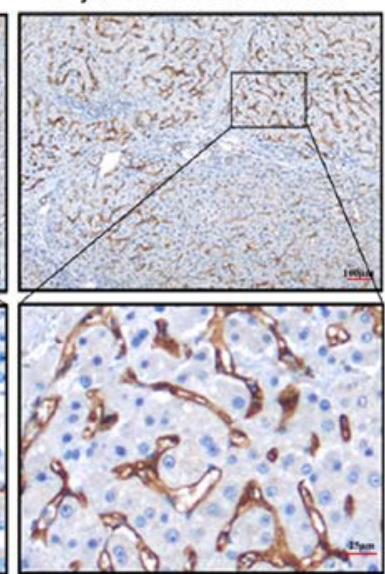

Normal hepatic tissue

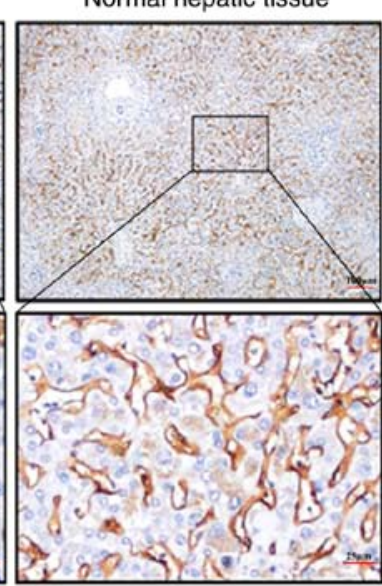

$\mathrm{C}$

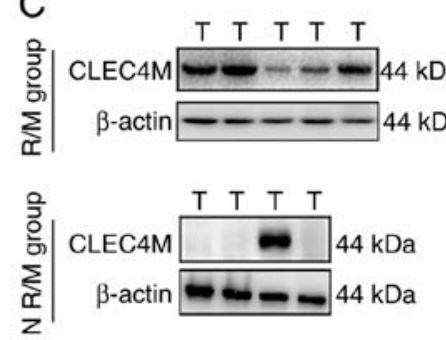

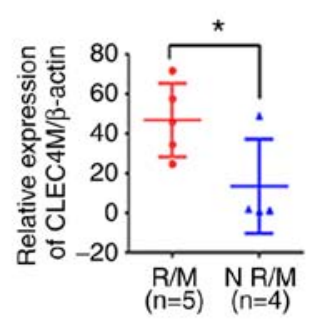

Figure 2. Association analysis between CLEC4M expression and HCC recurrence and metastasis. (A) Representative immunohistochemical staining of CLEC4M expression in R/M or N R/M HCC tumor tissues. (B) HCC tumor tissues in R/M group (n=59) displayed higher CLEC4M expression level comparing to N R/M group ( $n=29)$. (C) Western-blot analysis of CLEC4M expression in R/M group ( $=5$ ) and N R/M group ( $\mathrm{n}=4)$. * $\mathrm{P}<0.05$. R/M, recurrence/metastasis, $\mathrm{N} \mathrm{R/M}$, no recurrence/metastasis; T, tumor tissue; NT, non-tumor tissue. HCC, hepatocellular carcinoma; CLEC4M, C-type lectin domain family 4 member M.

in the moderate- and strong-positive group, and 26 months in negative- and weak-positive group. Additionally, the mean OS time was 29 months in the moderate- and strong-positive group, compared with 36 months in the negative- and weak-positive group. Subsequently, Cox's multivariate regression analysis was performed using recurrence/metastasis as the dependent variable. Age, sex, tumor size, tumor number, encapsulation, microvascular invasion, differentiation, AFP expression and disease stage were listed as independent variables. The results of the analysis revealed that high expression levels of CLEC4M independently predicted RFS $(\mathrm{P}=0.032)$ and OS times $(\mathrm{P}=0.011$; Table IV) of patients with HCC. The results of the present study suggest that CLEC4M is a promising prognostic biomarker for HCC, but a study conducted on a larger population size would improve the validity and accuracy of this hypothesis.

CLEC4M is specifically expressed in sinusoidal endothelial cells. Immunohistochemical staining illustrated that low-positive staining of CLEC4M was visualized in HCC tissues. The expression level of CLEC4M in HCC cells was also investigated. CD31 is a biomarker that is commonly used to label endothelial cells (27). Thus, co-localization staining, with CLEC4M and CD31 antibodies, was performed using the pathological tissue sections. The results of the current study indicated that CLEC4M is expressed in the same cells in which CD31-positive staining was observed (Fig. 4), which suggests that CLEC4M is specifically expressed in sinusoidal endothelial cells (but not in HCC cells), consistent with the results of a previous study (28).

\section{Discussion}

Tumor recurrence and metastasis are major contributors to the high mortality rate of patients with HCC (29). The discovery of sensitive and reliable biomarkers for the early diagnosis of HCC represents an effective strategy to reduce the rate of recurrence and metastasis, and improves prognosis. The clinical significance and biological function of CLEC4M have been well researched in colon (12) and gastric cancer (13). However, the influence that CLEC4M exerts on HCC progression is not yet well characterized. In the present study, CLEC4M expression was shown to be downregulated in HCC tissues compared with adjacent non-tumor tissues. Furthermore, the association between CLEC4M expression and certain clinicopathological features was investigated, including tumor size, extent of encapsulation, microvascular invasion, differentiation and tumor recurrence or metastasis. Higher CLEC4M expression in HCC tumor tissues correlated with shorter postoperative RFS and OS times in patients with HCC. Additionally, moderate- and strong-positive staining of CLEC4M was identified to be an independent prognostic indicator of RFS and OS in patients with HCC (following hepatectomy). The results of the present study systematically highlight CLEC4M as an effective biomarker that may predict the prognosis of patients with HCC.

CLEC4M is a C-type lectin that is primarily expressed in sinusoidal endothelial cells of the healthy liver, as demonstrated in the non-tumor section of Fig. 1E. Notably, CLEC4M 
Table II. Association between CLEC4M expression and certain clinicopathological features.

\begin{tabular}{|c|c|c|c|c|}
\hline \multirow[b]{2}{*}{ Clinicopathological feature } & \multirow[b]{2}{*}{ Patients, n (\%) } & \multicolumn{3}{|c|}{ Association with CLEC4M expression } \\
\hline & & Negative and weak (\%) & Moderate and strong (\%) & P-value \\
\hline Sex & & & & 0.984 \\
\hline Female & $6(6.82)$ & $4(66.7)$ & $2(33.3)$ & \\
\hline Male & $82(93.18)$ & $55(67.07)$ & $27(32.93)$ & \\
\hline Age, years & & & & 0.788 \\
\hline$\leq 50$ & $29(32.95)$ & $20(68.97)$ & $9(31.03)$ & \\
\hline$>50$ & $59(67.05)$ & $39(66.10)$ & $20(33.90)$ & \\
\hline HBsAg & & & & 0.668 \\
\hline Negative & $11(14.29)$ & $8(72.72)$ & $3(27.28)$ & \\
\hline Positive & $77(85.71)$ & $51(66.23)$ & $26(33.77)$ & \\
\hline $\mathrm{AFP}, \mathrm{ng} / \mathrm{ml}$ & & & & 0.408 \\
\hline$\leq 20$ & $40(45.45)$ & $25(62.50)$ & $15(37.50)$ & \\
\hline$>20$ & $48(54.55)$ & $34(70.83)$ & $14(29.17)$ & \\
\hline Liver cirrhosis & & & & 0.466 \\
\hline No & $32(36.36)$ & $23(78.88)$ & $9(21.12)$ & \\
\hline Yes & $56(63.64)$ & $36(64.29)$ & $20(35.71)$ & \\
\hline Tumor number & & & & 0.412 \\
\hline Single & $75(85.23)$ & $49(62.82)$ & $26(37.18)$ & \\
\hline Multiple & $13(14.77)$ & $10(76.92)$ & $3(23.08)$ & \\
\hline Tumor size, $\mathrm{cm}$ & & & & $0.018^{\mathrm{a}}$ \\
\hline$\leq 5$ & $52(59.09)$ & $40(81.13)$ & $12(18.87)$ & \\
\hline$>5$ & $36(40.91)$ & $19(52.78)$ & $17(47.22)$ & \\
\hline Tumor encapsulation & & & & $<0.001^{\mathrm{c}}$ \\
\hline Complete & $58(65.91)$ & $47(81.03)$ & $11(18.97)$ & \\
\hline None & $30(34.09)$ & $12(40.00)$ & $18(60.00)$ & \\
\hline Microvascular invasion & & & & $0.008^{\mathrm{b}}$ \\
\hline Absence & $42(47.73)$ & $34(80.95)$ & $8(19.05)$ & \\
\hline Present & $46(52.27)$ & $25(54.35)$ & $21(45.65)$ & \\
\hline TNM stage & & & & $0.019^{\mathrm{a}}$ \\
\hline $\mathrm{I}+\mathrm{II}$ & $46(52.27)$ & $36(78.26)$ & $10(21.74)$ & \\
\hline III+IV & $42(47.73)$ & $23(54.76)$ & $19(45.24)$ & \\
\hline BCLC stage & & & & 0.548 \\
\hline $0+\mathrm{A}$ & $70(79.54)$ & $48(68.57)$ & $22(31.43)$ & \\
\hline $\mathrm{B}+\mathrm{C}$ & $18(20.46)$ & $11(61.11)$ & $7(38.89)$ & \\
\hline HBV-DNA & & & & 0.703 \\
\hline Negative & $42(47.73)$ & $29(69.05)$ & $13(30.95)$ & \\
\hline Positive & $46(52.27)$ & $30(65.22)$ & $16(34.78)$ & \\
\hline
\end{tabular}

HBsAg, hepatitis B surface antigen; AFP, $\alpha$-fetoprotein; BCLC stage, Barcelona clinic liver cancer stage; HBV, hepatitis B virus; CLEC4M, C-type lectin domain family 4 member $\mathrm{M}$. ${ }^{\mathrm{P}} \mathrm{P}<0.05,{ }^{\mathrm{b}} \mathrm{P}<0.01,{ }^{\mathrm{c}} \mathrm{P}<0.001$.

expression has also been observed in the HCC tissues of patients that have experienced recurrence or metastasis. Additionally, high CLEC4M expression significantly correlated with microvascular invasion and tumor metastasis, which suggests that CLEC4M may promote angiogenesis. Multiple studies have provided indirect evidence supporting the present results. For example, Borentain et al (30) demonstrated that the inhibition of E-selectin suppressed hepatocellular carcinoma growth via the impairment of tumor angiogenesis. Moreover, DC-SIGN, which is highly homologous to CLEC4M, interacted with the Lewis $\mathrm{X}$ residues of carcinoembryonic antigen-related cell adhesion molecule 1 , resulting in angiogenesis $(31,32)$. Thus, an investigation into the influence of CLEC4M on the angiogenesis of HCC tissues should be performed in future experiments to further prove that CLEC4M play important roles in metastasis and invasion of HCC tissues. In summary, 
Table III. Immunohistochemical analysis of CLEC4M expression in 88 hepatocellular carcinoma tissues.

CLEC4M expression

\begin{tabular}{lcccr} 
Group & Patients, $\mathrm{n}(\%)$ & Moderate and strong $(\%)$ & Negative and weak $(\%)$ & P-value \\
\hline R/M & $54(61.36)$ & $24(44.44)$ & $30(55.56)$ & $0.004^{\mathrm{a}}$ \\
N R/M & $34(38.64)$ & $5(14.71)$ & $29(85.29)$ & \\
\hline
\end{tabular}

$\mathrm{R} / \mathrm{M}$, recurrence or metastasis, $\mathrm{N} \mathrm{R/M}$, no recurrence/metastasis; CLEC4M, C-type lectin domain family 4 member $\mathrm{M}$. ${ }^{\mathrm{a}} \mathrm{P}<0.01$.
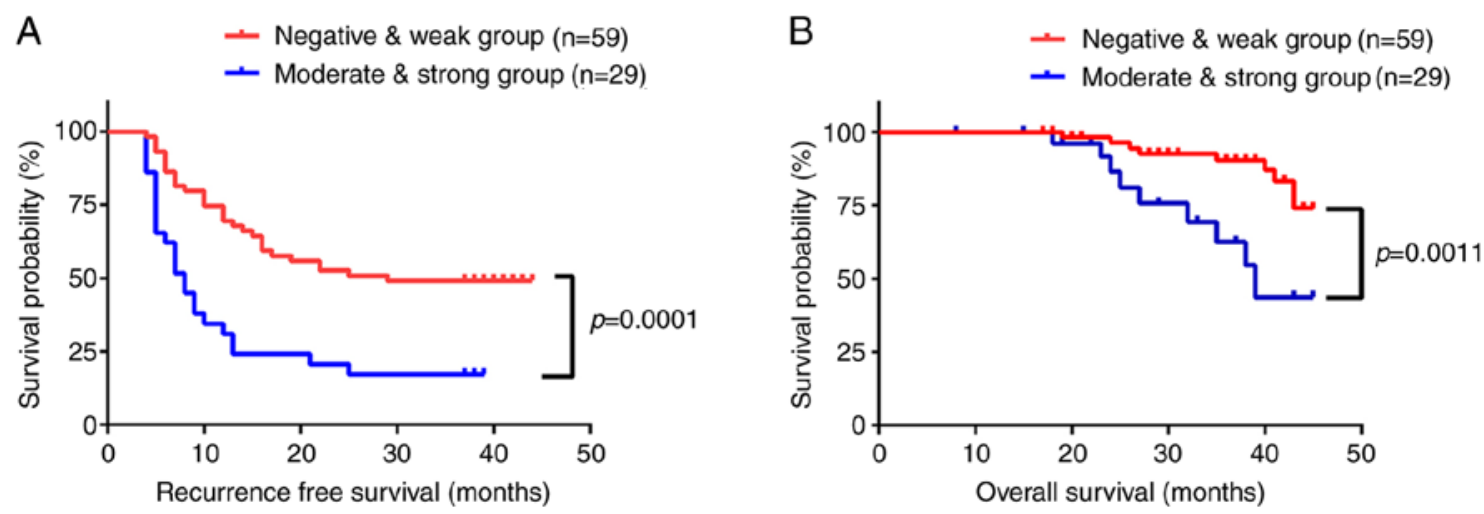

Figure 3. Kaplan-Meier analysis of the association between CLEC4M expression in HCC tumor tissues and the survival of patients with HCC. (A) Recurrence-free and (B) overall survival curves suggest that patients with moderate- or strong-positive CLEC4M staining exhibit higher recurrence rates ( $\mathrm{P}=0.0001)$ and shorter overall survival times $(\mathrm{P}=0.0011)$. These patients $(\mathrm{n}=29)$ had significantly higher recurrence rates and shorter overall survival times than those with negative or weak expression ( $\mathrm{n}=59$ ). HCC, hepatocellular carcinoma; CLEC4M, C-type lectin domain family 4 member M; RFS, recurrence free survival; OS, overall survival.
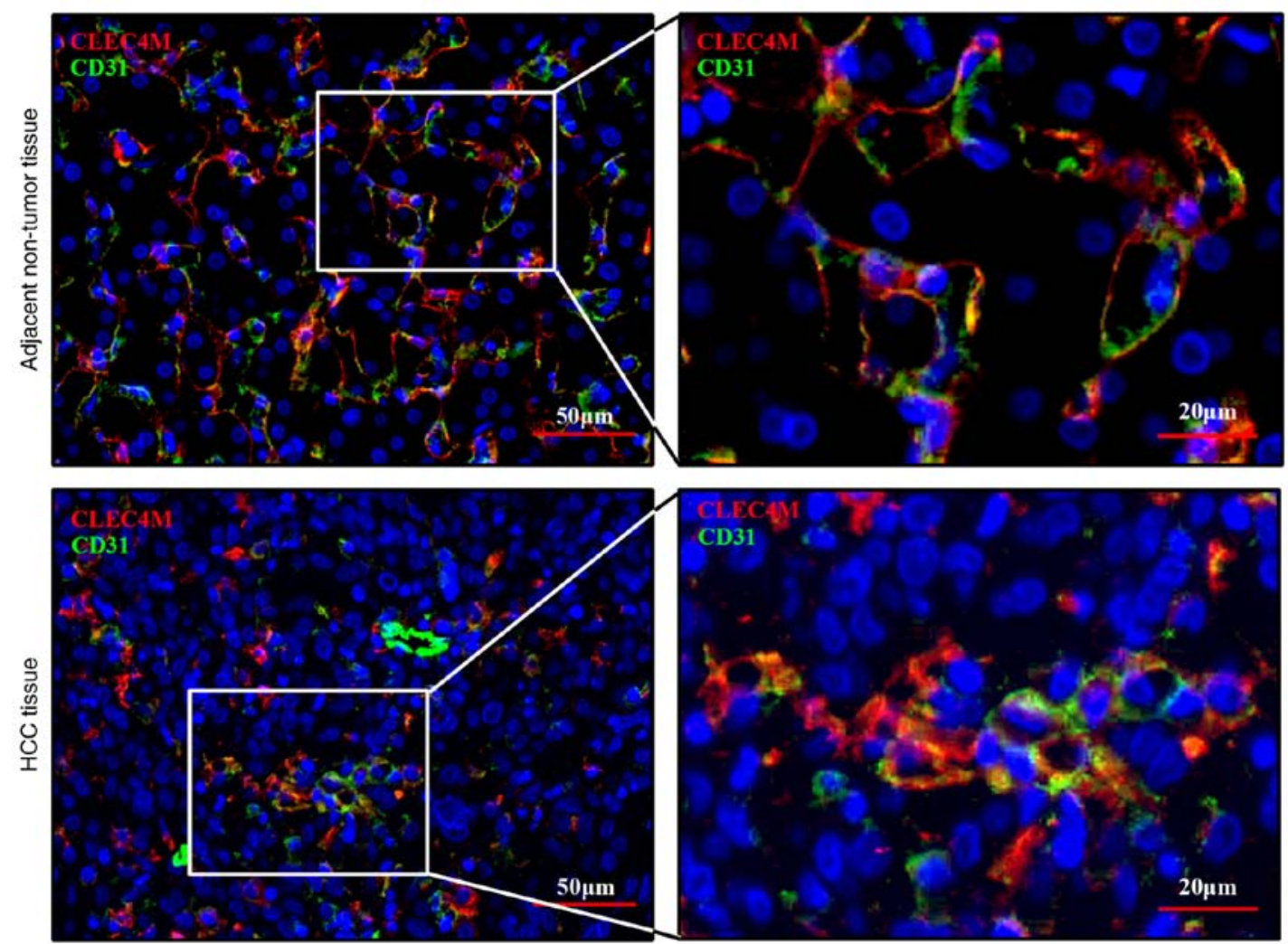

Figure 4. Co-localization analysis of CLEC4M and sinusoidal endothelial cells in HCC and adjacent non-tumor tissue. Co-staining of HCC tissue and adjacent non-tumor tissue for CLEC4M (red) and CD31 (green). Magnification, x200 and x400. HCC, hepatocellular carcinoma; CLEC4M, C-type lectin domain family 4 member $\mathrm{M}$. 


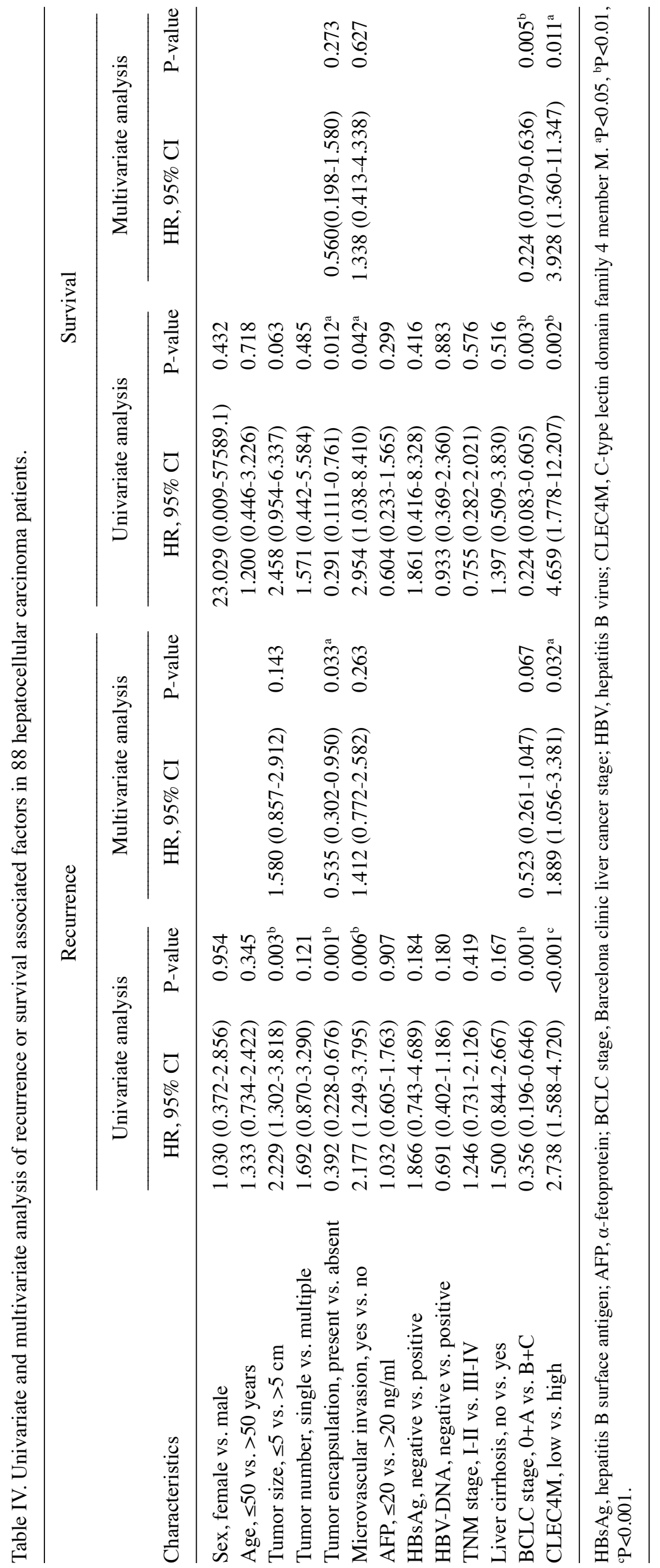


the present data indicate that CLEC4M is implicated in the progression of $\mathrm{HCC}$, in a similar manner to its association with colon and gastric cancer.

RT-qPCR determined that the expression of CLEC4M was significantly downregulated in tumor tissues, compared with non-tumor tissues. This appeared to contradict the fact that patients with HCC and high CLEC4M expression in tumor tissues typically exhibited shorter OS and RFS times. This may be attributable to the fact that CLEC4M was specifically expressed in sinusoidal endothelial cells, even in HCC tissues (Fig. 4), consistent with previous studies $(33,34)$. Additionally, in tissues containing many endothelial cells, the staining of CLEC4M appears stronger. Liver sinusoids consist of a line of sinusoidal endothelial liver cells and Kupffer cells, providing oxygen and nutrients to hepatocytes and forming a distribution network throughout the liver $(27,35,36)$. Additionally, CLEC4M can bind to intercellular adhesion molecule 3 (ICAM3; 28), resulting in the activation and recruitment of ICAM3-positive $\mathrm{T}$ cells and initiating an immune response to pathogens or cancer cells (37). Thus, a microenvironment with a low expression level of CLEC4M and incomplete microvasculature may favor early tumor development, in association with proliferation of tumor cells and escaping from immune surveillance in HCC cells. Subsequently, a gradual increase in the genesis of hepatic sinusoids and the surrounding vasculature may provide sufficient nutrition and oxygen proportional to the growth of the tumor, whilst allowing it time to adapt to the immune pressures of the host environment. Furthermore, it has been demonstrated that CLEC4M enhances the mobility and invasiveness of tumor cells in gastric and colon cancer $(13,21)$. Additionally, high CLEC4M expression in HCC tissues is associated with a poorer prognosis, which is consistent with previous literature on lung (38) and cervical cancer (39). Therefore, it is hypothesized that an increase in CLEC4M expression proportional to microvascular development may be beneficial to the growth and metastasis of HCC cells. This may also explain the correlation between moderate or strong-positive staining of CLEC4M in cancer tissues, and the high risk of recurrence and metastasis.

In conclusion, the current study demonstrated that expression levels of CLEC4M in HCC tissues may be an effective indicator of HCC progression, and may represent a potential target for therapeutic development.

\section{Acknowledgements}

The authors would like to thank Dr Bin Wang and Dr Mojiao Liu, The Department of Pathology, Mengchao Hepatobiliary Hospital of Fujian Medical University, for their technical help. The results shown in this study are in part based upon data generated by the TCGA Research Network: https://www. cancer.gov/tcga.

\section{Funding}

The present study was supported by The National Natural Science Foundation of China (grant no. 81602102 and grant no. 81672376), the Natural Science Foundation of Fujian Province (grant no. 2016J01417 and 2017J01266), the Young and Middle-aged Talent Training Project of Fujian provincial health and Family Planning Commission (grant no. 2018-ZQN-76; 2018-ZQN-37; and 2016-1-44), the Joint Funds for the Innovation of Science and Technology of Fujian province (grant no. 2017Y9116 and 2017Y9041), the Scientific Foundation of Fuzhou City (grant no. 2015-S-143-19) and the Startup Fund for scientific research, Fujian Medical University (grant no. 2018QH1194).

\section{Availability of data and materials}

The datasets used and/or analyzed during the present study are available from the corresponding author on reasonable request.

\section{Authors' contributions}

XLL, YCW and JFL were responsible for research creation and design, and provided study material. LHC provided the pathological sections and perform the immunochemical score together with LPL. LPL, KK, BXZ, LLW, CLZ and FW collected, assembled the clinical and follow-up data. NSL and XYZ analysed and interpreted the data. LPL and YCW drafted and finalized the manuscript. All authors read and approved the final version of the manuscript.

\section{Ethics approval and consent to participate}

All the experiments were approved by the Ethics committee of Mengchao Hepatobiliary Hospital of Fujian Medical University (Fujian, China).

\section{Patient consent for publication}

Not applicable.

\section{Competing interests}

The authors declare that they have no competing interests.

\section{References}

1. Bray F, Ferlay J, Soerjomataram I, Siegel RL, Torre LA and Jemal A: Global cancer statistics 2018: GLOBOCAN estimates of incidence and mortality worldwide for 36 cancers in 185 countries. CA Cancer J Clin 68: 394-424, 2018.

2. Zhu ZX, Huang JW, Liao MH and Zeng Y: Treatment strategy for hepatocellular carcinoma in China: Radiofrequency ablation versus liver resection. Jpn J Clin Oncol 46: 1075-1080, 2016.

3. Zhao LY, Huo RR, Xiang X, Torzilli G, Zheng MH, Yang T, Liang XM, Huang X, Tang PL, Xiang BD, et al: Hepatic resection for elderly patients with hepatocellular carcinoma: A systematic review of more than 17,000 patients. Expert Rev Gastroenterol Hepatol 12: 1059-1068, 2018.

4. Zhao Y, Wang M, Cui C, Zhang L, Liao F, Li H and Wu X: Significance of combined tests of serum golgi glycoprotein 73 and other biomarkers in diagnosis of small primary hepatocellular carcinoma. Cancer Biomark 15: 677-683, 2015.

5. Johnson PJ: The role of serum alpha-fetoprotein estimation in the diagnosis and management of hepatocellular carcinoma. Clin Liver Dis 5: 145-159, 2001.

6. Amr KS, Elmawgoud Atia HA, Elazeem Elbnhawy RA and Ezzat WM: Early diagnostic evaluation of miR-122 and miR-224 as biomarkers for hepatocellular carcinoma. Genes Dis 4: 215-221, 2017.

7. Ding D, Yao Y, Zhang S, Su C and Zhang Y: C-type lectins facilitate tumor metastasis. Oncol Lett 13: 13-21, 2017.

8. Dambuza IM and Brown GD: C-type lectins in immunity: Recent developments. Curr Opin Immunol 32: 21-27, 2015. 
9. Ferroni P, Roselli M, Spila A, D'Alessandro R, Portarena I, Mariotti S, Palmirotta R, Buonomo O, Petrella G and Guadagni F: Serum sE-selectin levels and carcinoembryonic antigen mRNA-expressing cells in peripheral blood as prognostic factors in colorectal cancer patients. Cancer 116: 2913-2921, 2010.

10. Guo RM, Zhao CB, Li P, Zhang L, Zang SH and Yang B: Overexpression of CLEC18B associates with the proliferation, migration, and prognosis of glioblastoma. ASN Neuro 10 1759091418781949,2018

11. van Gisbergen KP, Aarnoudse CA, Meijer GA, Geijtenbeek TB and van Kooyk Y: Dendritic cells recognize tumor-specific glycosylation of carcinoembryonic antigen on colorectal cancer cells through dendritic cell-specific intercellular adhesion molecule-3-grabbing nonintegrin. Cancer Res 65: 5935-5944, 2005.

12. Zuo Y, Ren S, Wang M, Liu B, Yang J, Kuai X, Lin C, Zhao D Tang L and He F: Novel roles of liver sinusoidal endothelial cell lectin in colon carcinoma cell adhesion, migration and in-vivo metastasis to the liver. Gut 62: 1169-1178, 2013.

13. Zhang Y, Zhang Q, Zhang $M$, Yuan $M$, Wang Z, Zhang J, Zhou X, Zhang Y, Lin F, Na H, et al: DC-SIGNR by influencing the lncRNA HNRNPKP2 upregulates the expression of CXCR4 in gastric cancer liver metastasis. Mol Cancer 16: 78, 2017.

14. Coupland LA, Chong BH and Parish CR: Platelets and P-selectin control tumor cell metastasis in an organ-specific manner and independently of NK cells. Cancer Res 72: 4662-4671, 2012.

15. Yasmin-Karim S, King MR, Messing EM and Lee YF: E-selectin ligand-1 controls circulating prostate cancer cell rolling/adhesion and metastasis. Oncotarget 5: 12097-12110, 2014.

16. Pohlmann S, Soilleux EJ, Baribaud F, Leslie GJ, Morris LS, Trowsdale J, Lee B, Coleman N and Doms RW: DC-SIGNR, a DC-SIGN homologue expressed in endothelial cells, binds to human and simian immunodeficiency viruses and activates infection in trans. Proc Natl Acad Sci USA 98: 2670-2675, 2001.

17. Soilleux EJ, Morris LS, Leslie G, Chehimi J, Luo Q, Levroney E, Trowsdale J, Montaner LJ, Doms RW, Weissman D, et al: Constitutive and induced expression of DC-SIGN on dendritic cell and macrophage subpopulations in situ and in vitro. J Leukoc Biol 71: 445-457, 2002

18. da Silva RC, Segat L, Zanin V, Arraes LC and Crovella S Polymorphisms in DC-SIGN and L-SIGN genes are associated with HIV-1 vertical transmission in a Northeastern Brazilian population. Hum Immunol 73: 1159-1165, 2012.

19. Chen PC, Chuang PK, Chen CH, Chan YT, Chen JR, Lin SW, Ma C, Hsu TL and Wong CH: Role of N-linked glycans in the interactions of recombinant $\mathrm{HCV}$ envelope glycoproteins with cellular receptors. ACS Chem Biol 9: 1437-1443, 2014.

20. Op den Brouw ML, de Jong MA, Ludwig IS, van der Molen RG, Janssen HL, Geijtenbeek TB and Woltman AM: Branched oligosaccharide structures on HBV prevent interaction with both DC-SIGN and L-SIGN. J Viral Hepat 15: 675-683, 2008

21. Na H, Liu X, Li X, Zhang X, Wang Y, Wang Z, Yuan M, Zhang Y Ren S and Zuo Y: Novel roles of DC-SIGNR in colon cancer cell adhesion, migration, invasion, and liver metastasis. J Hematol Oncol 10: 28, 2017.

22. Cai Z, Zeng Y, Xu B, Gao Y, Wang S, Zeng J, Chen L, Huang A, Liu $X$ and Liu J: Galectin-4 serves as a prognostic biomarker for the early recurrence/metastasis of hepatocellular carcinoma. Cancer Sci 105: 1510-1517, 2014.

23. Remmele W, Hildebrand U, Hienz HA, Klein PJ, Vierbuchen M, Behnken LJ, Heicke B and Scheidt E: Comparative histological, histochemical, immunohistochemical and biochemical studies on oestrogen receptors, lectin receptors, and Barr bodies in human breast cancer. Virchows Arch A Pathol Anat Histopathol 409: 127-147, 1986.

24. Livak KJ and Schmittgen TD: Analysis of relative gene expression data using real-time quantitative PCR and the 2(-Delta Delta C(T)) method. Methods 25: 402-408, 2001.

25. Dobin A, Davis CA, Schlesinger F, Drenkow J, Zaleski C, Jha S, Batut P, Chaisson M and Gingeras TR: STAR: Ultrafast universal RNA-seq aligner. Bioinformatics 29: 15-21, 2013.
26. Speir ML, Zweig AS, Rosenbloom KR, Raney BJ, Paten B, Nejad P, Lee BT, Learned K, Karolchik D, Hinrichs AS, et al: The UCSC Genome Browser database: 2016 update. Nucleic Acids Res 44: D717-D725, 2016.

27. Crouch EE and Doetsch F: FACS isolation of endothelial cells and pericytes from mouse brain microregions. Nat Protoc 13: 738-751, 2018.

28. Lai WK, Sun PJ, Zhang J, Jennings A, Lalor PF, Hubscher S, McKeating JA and Adams DH: Expression of DC-SIGN and DC-SIGNR on human sinusoidal endothelium: A role for capturing hepatitis C virus particles. Am J Pathol 169: 200-208, 2006.

29. Chen Y, Gao SG, Chen JM, Wang GP, Wang ZF, Zhou B, Jin CH, Yang YT and Feng XS: Risk factors for the long-term efficacy, recurrence, and metastasis in small hepatocellular carcinomas. Cell Biochem Biophys 72: 627-631, 2015.

30. Borentain P, Carmona S, Mathieu S, Jouve E, El-Battari A and Gerolami R: Inhibition of E-selectin expression on the surface of endothelial cells inhibits hepatocellular carcinoma growth by preventing tumor angiogenesis. Cancer Chemother Pharmacol 77: 847-856, 2016.

31. Bogoevska V, Horst A, Klampe B, Lucka L, Wagener C and Nollau P: CEACAM1, an adhesion molecule of human granulocytes, is fucosylated by fucosyltransferase IX and interacts with DC-SIGN of dendritic cells via Lewis $\mathrm{x}$ residues. Glycobiology 16: 197-209, 2006.

32. Koch AE, Halloran MM, Haskell CJ, Shah MR and Polverini PJ: Angiogenesis mediated by soluble forms of E-selectin and vascular cell adhesion molecule-1. Nature 376: 517-519, 1995.

33. Bashirova AA, Geijtenbeek TB, van Duijnhoven GC, van Vliet SJ, Eilering JB, Martin MP, Wu L, Martin TD, Viebig N, Knolle PA, et al: A dendritic cell-specific intercellular adhesion molecule 3-grabbing nonintegrin (DC-SIGN)-related protein is highly expressed on human liver sinusoidal endothelial cells and promotes HIV-1 infection. J Exp Med 193: 671-678, 2001.

34. Falkowska E, Durso RJ, Gardner JP, Cormier EG, Arrigale RA, Ogawa RN, Donovan GP, Maddon PJ, Olson WC and Dragic T: L-SIGN (CD209L) isoforms differently mediate trans-infection of hepatoma cells by hepatitis C virus pseudoparticles. J Gen Virol 87: 2571-2576, 2006.

35. Brunt EM, Gouw AS, Hubscher SG, Tiniakos DG, Bedossa P, Burt AD, Callea F, Clouston AD, Dienes HP, Goodman ZD, et al: Pathology of the liver sinusoids. Histopathology 64: 907-920, 2014.

36. Patten DA, Wilson GK, Bailey D, Shaw RK, Jalkanen S, Salmi M, Rot A, Weston CJ, Adams DH and Shetty S: Human liver sinusoidal endothelial cells promote intracellular crawling of lymphocytes during recruitment: A new step in migration. Hepatology 65: 294-309, 2017.

37. Koppel EA, van Gisbergen KP, Geijtenbeek TB and van Kooyk Y: Distinct functions of DC-SIGN and its homologues L-SIGN (DC-SIGNR) and mSIGNR1 in pathogen recognition and immune regulation. Cell Microbiol 7: 157-165, 2005.

38. Liu X, Zhang H, Su L, Yang P, Xin Z, Zou J, Ren S and Zuo Y: Low expression of dendritic cell-specific intercellular adhesion molecule-grabbing nonintegrin-related protein in lung cancer and significant correlations with brain metastasis and natural killer cells. Mol Cell Biochem 407: 151-160, 2015.

39. Wang X, Jiang Y, Yuan M, Chen C, Wang K, Zhang Q, Zuo Y and Ren S: Overexpression of dendritic cell-specific intercellular adhesion molecule-3-grabbing nonintegrin-related protein in cervical cancer and correlation with squamous cell carcinoma antigen. Oncol Lett 14: 2813-2821, 2017.

This work is licensed under a Creative Commons Attribution-NonCommercial-NoDerivatives 4.0 International (CC BY-NC-ND 4.0) License. 